\title{
Avaliação dos erros de diluição de medicamentos de administração intravenosa em ambiente hospitalar para o desenvolvimento de um guia de diluição e administração dos mesmos
}

\author{
Errors on dilution of intravenous medication in a hospital, and \\ development of a drug dilution and administration guide
}

Recebido em: 04/02/2016

Aceito em: 26/05/2016
Danyelle Cristine MARINI; Juliana Talita PINHEIRO;

Claudia Solano ROCHA

Faculdades Integradas Maria Imaculada Rua Paula Bueno, 240,

Centro. Mogi Guaçu, SP. CEP 13840050, Brasil.

E-mail:danymarini@gmail.com

\begin{abstract}
The adverse events from medication are all kind of damage caused to patients, that can lead to significant harm to health, with relevant economic and social consequences, which is considered a public health problem. The medication errors, as an adverse event, can be avoided. On a hospital environment, errors on medication are a responsibility of the nursing team, due to its direct relation to the patient. Previous studies have shown that the frequency of the errors varies from one to six doses given, with different justifications. The study aimed to identify the errors frequency on the dilution of intravenous medication process, to propose an action that helps its reduction. The data research was made in October 2014, typified as a transversal descriptive research, with an observation nature. During this period, the activities of 18 nursing technicians involved in the intravenous medication preparation and administration, who worked in Intensive Therapy Units, were observed. 180 doses were verified, from which 125 (69.5\%) presented at least one dilution error. In 90 doses, (72\%) it was noticed more than one error per dose, and no evaluation or report of the medication errors was made. It was noticed that the professionals involved with the errors did not know how to identify them, and such event may compromise the effectiveness of the treatment. Also, the medications with a higher frequency of dilution errors were identified. Therefore, other diluents were defined, according to specific literature. This outcome turned into an educational material, as a file, to be quickly consulted.
\end{abstract}

Keywords: medication errors, hospital pharmacy, adverse events

\section{RESUMO}

Os eventos adversos em medicamentos são quaisquer danos causados ao paciente, que podem levar a importantes agravos à saúde, com relevantes repercussões econômicas e sociais, sendo considerado um problema de saúde pública. No âmbito hospitalar, erros de administração de medicamentos são atribuídos à equipe de enfermagem, devido à sua atuação direta com o paciente. O estudo teve como objetivo identificar a frequência de erros ocorridos no processo de diluição de medicamentos intravenosos, a fim de propor estratégias que colaborassem para a sua minimização. A coleta de dados ocorreu em outubro de 2014, e se caracterizou como pesquisa descritiva transversal, de natureza observacional. Durante este período, foi observado o trabalho de 18 técnicos de enfermagem, atuantes em unidade de terapia intensiva (UTI), envolvidos no processo de preparo e administração de medicamentos intravenosos. 180 doses foram observadas, das quais 125 (69,5\%) apresentaram pelo menos um erro de diluição; em 90 doses (72\%), ocorreu mais de um erro por dose, e não foi detectada nenhuma forma de registro nem avaliação e/ou monitoramento dos erros de medicação. Constatou-se que os profissionais envolvidos com os erros de medicação não souberam identificá-los, e este tipo de evento pode vir a comprometer a eficácia terapêutica do medicamento em questão. Também foram identificados os medicamentos mais prevalentes envolvidos nos erros de diluição, para os quais se estipulou o(s) diluente(s) mais adequado(s) conforme literatura consultada. Este resultado foi transformado em material didático-instrucional, em forma de fichário, para consulta rápida.

Palavras-chave: erros de medicação, farmácia hospitalar, eventos adversos 


\section{INTRODUÇÃO}

Nas últimas décadas, a segurança do paciente em ambiente hospitalar vem recebendo enfoque e gerado debates a nível mundial, com várias interpretações; entre elas, a de que segurança consiste na redução do risco e danos desnecessários, associados à assistência em saúde, até um mínimo aceitável. O mínimo aceitável refere-se àquilo que é viável diante do conhecimento atual, dos recursos disponíveis e do contexto em que a assistência foi realizada frente ao risco de não tratamento, ou outro tratamento. Entre os recursos disponíveis, o medicamento é um dos mais utilizados, no entanto, eventos adversos e erros relacionados a medicamentos são frequentes no ambiente hospitalar (1).

Segundo o National Coordinating Council for Medication Error Reporting and Prevention (NCC MERP) - uma corporação norte-americana fundada para disseminar conhecimento, estimular relatos e prevenir erros - erro de medicação pode ser definido como qualquer evento evitável que pode causar ou induzir o uso inapropriado de medicamento ou prejudicar o paciente enquanto o medicamento está sob o controle do profissional de saúde, paciente ou consumidor. Tais eventos podem estar relacionados à prática profissional, produtos para saúde, procedimentos, e sistemas, incluindo prescrição, comunicação, etiquetação, embalagem e nomenclatura; aviamento; dispensação; distribuição; administração; educação; monitoramento e uso $(2,3)$.

Os tipos de erros descritos tanto na literatura nacional como na internacional são: erros de prescrição de medicamento e/ou de transcrição, dispensação, administração de um medicamento diferente do prescrito; erros de omissão de dose ou do medicamento; erros quanto à dose e forma farmacêutica; erro de preparo, manipulação e/ou acondicionamento; erro de frequência, técnica, via, velocidade e horário de administração; erro de paciente; duração e monitorização insuficientes do tratamento; uso de medicamento deteriorado; falta de adesão do paciente $(2,4)$.

Os erros envolvendo medicamentos podem ou não causar danos aos pacientes; estudos ao longo dos últimos anos mostraram que a incidência destes erros causados pela equipe de enfermagem, varia de um erro a cada seis doses administradas; os quais se justificam por diferentes mecanismos (5).

As principais causas de erros de medicação foram atribuídas à má qualidade da grafia médica, aos diferentes sistemas de pesos e medidas adotados no mesmo hospital, à utilização de abreviaturas não padronizadas, aos medicamentos com nomes comerciais semelhantes, às ordens médicas verbais, às informações médicas incompletas e confusas, às múltiplas transcrições de prescrições, às falhas de comunicação para as suspensões de medicamentos, às interpretações de dosagens, aos problemas no ambiente de preparo das medicações, ao acúmulo de atividades, entre outras (5). Este tipo de erro vem representando uma triste realidade para os pacientes, profissionais e instituições hospitalares, pois suas consequências repercutem nos indicadores de qualidade da assistência em saúde (2).

A prática de medicação em uma organização hospitalar pode ser definida como um sistema complexo, com vários processos interligados, interdependentes $\mathrm{e}$ constituído por profissionais de diferentes áreas do conhecimento (médicos, equipe de farmácia e de enfermagem) que compartilham de um objetivo comum, que é a prestação da assistência à saúde dos pacientes com qualidade, eficácia e segurança (6).

De acordo com a Joint Commission on Accreditation of Healthcare Organizations (JCAHO), o sistema de medicação pode ser dividido em cinco processos: seleção e obtenção do medicamento, prescrição e/ ou transcrição, preparo e dispensação, administração de medicamentos e monitoramento do paciente em relação aos efeitos do medicamento $(3,7)$.

Quanto ao preparo de medicamentos, processo no qual se realiza a diluição, devem ser aplicados vários princípios científicos que garantam o resultado terapêutico esperado. Para isto, é necessário saber com o que diluir, qual o volume a ser administrado em acesso venoso periférico e/ou central, quanto tempo a medicação pode permanecer preparada antes de ser administrada, em que condições ambientais deve ser preparada e acondicionada (luz, calor, higiene), além de aspectos tradicionalmente vinculados à enfermagem, como garantir o preparo do medicamento adequado ao paciente certo e na dose correta (1).

Em relação à frequência de erros, em cada um dos processos, um estudo apontou que $39 \%$ dos erros ocorreram na prescrição de medicamentos, $12 \%$ na transcrição, $11 \%$ na dispensação, e $38 \%$ no preparo e administração de medicamentos (3).

Segundo Silva e Cassiani (2004), os tipos de erros relacionados à medicação, citados pelos participantes, foram os relacionados à prescrição de medicamentos com ( $29 \%$ dos resultados), seguidos dos erros relacionados ao horário $(20,6 \%)$, e ao preparo e administração dos medicamentos $(13,6 \%)(2)$.

Embora em nosso país não existam estudos suficientes que mostrem a incidência de erro, sabemos que 
no ranking mundial de consumo de medicamentos o Brasil assume a quinta posição (8); e que pesquisas sobre eventos adversos, neles incluídos os erros de prescrição, dispensação e administração, têm avançado (1). Assim, a assistência segura e isenta de riscos ou de danos à clientela deve ser meta da equipe multidisciplinar, que deve sempre estar amparada no conhecimento científico, nas normas legais que regulamentam os direitos e obrigações relativas ao exercício profissional, assim como na dimensão ética e moral que permeiam suas ações (9).

Nas unidades hospitalares, a segurança na terapia medicamentosa merece enfoque especial, visto que a combinação de múltiplos fármacos, gravidade e instabilidade dos pacientes e, às vezes, a total dependência dos mesmos em relação à equipe multidisciplinar, são fatores que predispõem o paciente a uma maior vulnerabilidade (10).

Diante do exposto, o presente estudo teve como objetivo identificar a frequência de erros ocorridos no processo de preparo e administração de medicamentos intravenosos, mais precisamente à diluição destes medicamentos, a fim de propor estratégias que colaborem para a minimização destes erros.

\section{MATERIAIS E MÉTODOS}

A pesquisa foi realizada em um hospital de administração pública, localizado em um município no interior do Estado de São Paulo. Este se caracteriza por ser um hospital geral, de pequeno porte, com capacidade para atender até 50 leitos, distribuídos entre unidade ambulatorial, clínica cirúrgica, clínica médica e unidade de terapia intensiva (UTI).

A população em estudo foi composta por profissionais de enfermagem, de nível técnico médio, que participaram do processo de preparo e administração de medicamentos intravenosos, pertencentes ao quadro de funcionários do hospital em estudo, atuantes em UTI. A escolha desta unidade ocorreu devido à presença de pacientes críticos que fazem uso de múltiplos fármacos.

Aqueles que atenderam aos critérios de inclusão e concordaram em participar da pesquisa de forma voluntária foram observados quanto à conduta no preparo e à administração de medicamentos intravenosos, mediante assinatura de Termo de Consentimento Livre e Esclarecido. O projeto foi aprovado pelo Comitê de Ética das Faculdades Integradas Maria Imaculada conforme protocolo ${ }^{0}$ 126/2014.

A coleta de dados ocorreu durante o mês de outubro de 2014, e se caracterizou como uma pesquisa descritiva transversal, de natureza observacional. Os dados coletados foram tabulados e, posteriormente, analisados de acordo com a literatura consultada.

A análise literária foi produto de uma investigação sobre o tema "diluição e administração de medicamentos intravenosos", realizada em bancos de dados nacionais e internacionais, como Scielo, Bireme, Lilacs, Medscape, Micromedex, etc. Na busca, foram utilizadas expressões como: administração intravenosa de medicamentos, erros de medicação, segurança em terapia medicamentosa. Os critérios de seleção incluíram referências que contribuíam para o cumprimento dos objetivos da pesquisa, sendo consideradas as publicações datadas de 2000 a 2014, nos idiomas português, inglês e espanhol. Foram excluídos artigos sem disponibilidade integral do conteúdo, e artigos duplicados. Também foram consultados livros, que continham como temática principal a prática da farmácia hospitalar e farmácia clínica; manuais de diluição de medicamentos e, bulas dos medicamentos envolvidos no estudo. Como resultado disto elaborou-se o material didático-instrucional para consulta rápida.

\section{RESULTADOS E DISCUSSÃO}

Caracterização da população em estudo. A população do presente estudo foi constituída por 18 técnicos de enfermagem (90\%) do total existentes na UTI, campo de estudo; e caracterizou-se por apresentar perfil em maior parte feminino (89\%) (Figura 1).

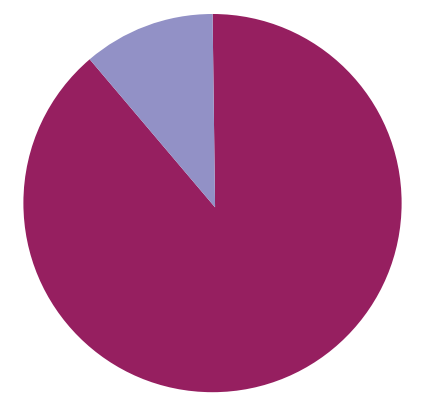

\section{- Feminino Masculino}

Figura 1 - Profissionais de Enfermagem na Unidade de Tratamento Intensivo em um hospital do interior de São Paulo, Brasil (outubro de 2014)

Quanto à caracterização, segundo a faixa etária, a equipe de enfermagem apresentou maior porcentagem entre 30 e 35 anos (33\%), seguida por aqueles com idade entre 40 e 45 anos (22\%). sendo a idade mínima de 23 anos e a máxima de 54 anos (Figura 2). A maioria dos profissionais de enfermagem participantes apresentou tempo de formado igual a 5 anos (39\%), sendo a minoria com tempo de formado superior a 5 anos $(28 \%)$ 


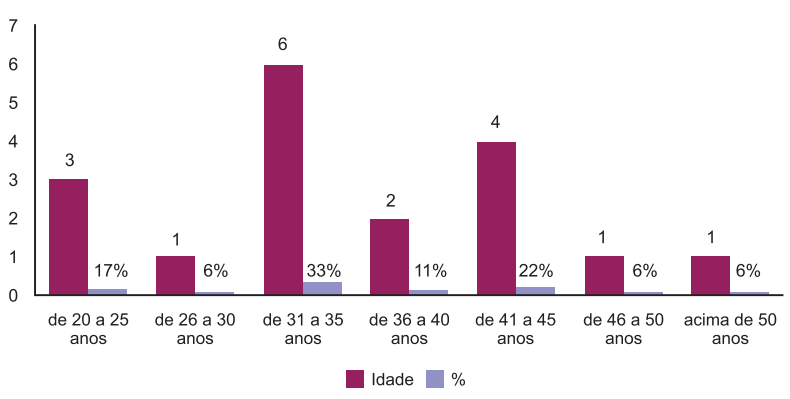

Figura 2 - Faixa etária dos profissionais de Enfermagem na Unidade de Tratamento Intensivo em um hospital do interior de São Paulo, Brasil (outubro de 2014)

O tempo de experiência profissional em UTI foi, para a maioria dos entrevistados, inferior a 5 anos (55\%), seguidos por experiência de 5 anos (27\%), enquanto apenas $18 \%$ dos profissionais afirmaram ter experiência profissional em UTI superior a 5 anos.

Identificação dos erros de diluição de medicamentos intravenosos. Para a avaliação dos erros de medicação envolvendo o processo de diluição, foi considerado como erro a não prescrição do diluente, a prescrição de diluente inadequado, o uso de diluente inadequado, o não uso de diluente, a associação de medicamentos incompatíveis em seringas, a associação de medicamentos incompatíveis em soluções e, a infusão em Y de medicamentos incompatíveis.

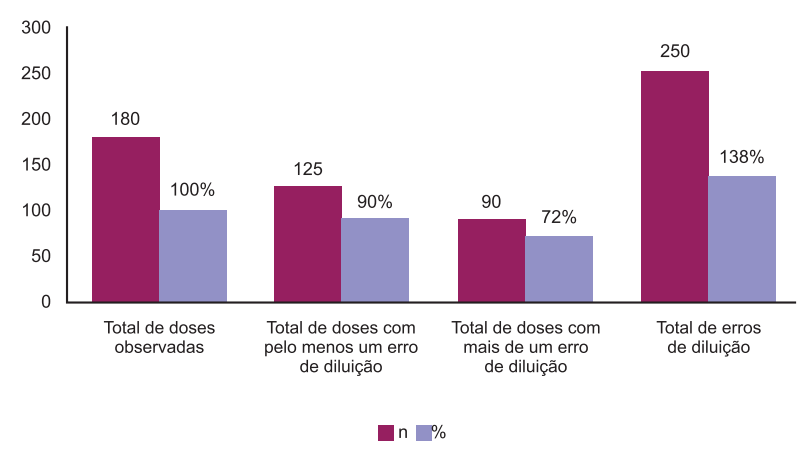

Figura 3 - Prevalência de erro de diluição medicamentosa das doses medicamentosas observada em Unidade de Tratamento Intensivo de um hospital do interior de São Paulo, Brasil (outubro de 2014)

Com relação a esta última categoria, vale ressaltar que os dispositivos intravenosos mais comumente utilizados na infusão de medicamentos intravenosos são os cateteres periféricos e dispositivos de infusão múltipla, que apresentam duas ou quatro vias, e permitem a infusão simultânea de soluções compatíveis em um mesmo acesso venoso (infusão em $\mathrm{Y}$ ) quando conectados ao cateter periférico. Porém, nem sempre é observada a compatibilidade das soluções que serão infundidas de forma concomitante (11).

Durante o período da pesquisa, foram observadas a manipulação de 10 doses medicamentosas por profissional, totalizando 180 doses, das quais $125(69,5 \%)$ apresentaram pelo menos um erro de diluição. Dessas, foram observados mais de um erro por dose em 90 doses (72\%), totalizando 250 erros de diluição de medicamentos intravenosos (Figura 3).

Dentre os erros avaliados, aquele envolvendo a infusão em Y de medicamento incompatível (32\%) mostrou ser o mais frequente; seguido pelos erros referentes a não prescrição de diluente $(24 \%)$ e ao uso de diluente inadequado (16\%). O não uso de diluente e a associação de medicamentos incompatíveis em seringa foram os erros com igual e menor incidência (4\%) (Tabela 1).

Tabela 1: Erros de diluição de medicamentos intravenosos observados em Unidade de Tratamento Intensivo de um hospital do interior de São Paulo, Brasil (outubro de 2014).

\begin{tabular}{|c|c|c|}
\hline Categorias de erro & $\mathbf{n}$ & $\%$ \\
\hline Prescrição inadequada de diluente & 30 & 12 \\
\hline Não prescrição de diluente & 60 & 24 \\
\hline Uso de diluente inadequado & 40 & 16 \\
\hline Não uso de diluente & 10 & 4 \\
\hline Associação em seringa de medicamentos incompatíveis & 20 & 8 \\
\hline Associação em solução de medicamentos incompatíveis & 10 & 4 \\
\hline Infusão em Y de medicamento incompatível & 80 & 32 \\
\hline Total & 250 & 100 \\
\hline
\end{tabular}


Identificação dos medicamentos envolvidos nos erros de diluição. Na Tabela 2 é apresentado o levantamento dos medicamentos prevalentes e relacionados com os erros de diluição. No geral, os fármacos que apresentaram maior índice de erros de diluição foram a fenitoína e a norepinefrina (12,8\%). Na categoria prescrição inadequada de diluente, a norepinefrina foi o fármaco prevalente $(5,2 \%)$, enquanto a mesma prevalência foi encontrada para a fentanila, quanto aos erros envolvendo a não utilização de diluente; a metoclopramida para os erros de associação em seringa com medicamento incompatível; e a dipirona para os erros de infusão em Y com medicamento incompatível.

Os erros envolvendo a fenitoína foram aqueles envolvidos com o uso de diluente inadequado $(2,8 \%) \mathrm{e}$ associação deste em solução incompatível (2,4\%).

Elaboração do material didático-instrucional. O material didático-instrucional foi elaborado conforme a caracterização preliminar do objeto de estudo (medicamentos prevalentes nos erros de diluição). Foi constituído em forma de fichário, visando a aplicabilidade e praticidade da utilização desses impressos na área de manipulação de injetáveis, buscando assim a melhor maneira para aperfeiçoar a busca das informações com maior rapidez. O resultado deste estudo encontra-se no Quadro 1.

A população em estudo apresentou predominância feminina $(90 \%)$ e faixa etária prevalente entre 30 e 35 anos $(33,33 \%)$, dados estes que corroboram com outros estudos $(7,9,18)$. Para Lopes e Leal $(2005)$, a hegemonia feminina na prestação de cuidados de saúde deve-se à seletividade exercida pelo ensino e mercado de trabalho, além da histórica condição das mulheres como "cuidadoras", na qual o cuidar é, de certa forma, uma ação feminina que transcende o espaço de trabalho (19).

Com relação ao tempo de formação, a maioria do grupo em estudo apresentou até 5 anos (72\%), e o tempo de experiência em UTI foi inferior a 5 anos (55\%). Este resultado coincide com outro estudo no qual a justificativa apresentada para tal resultado foi o forte atrativo que as UTI exercem sobre os profissionais recém-formados, que veem nelas a possibilidade de prestar assistência direta ao paciente, além da oportunidade de desenvolverem habilidades técnico-científicas (18).

Tabela 2: Relação dos erros de diluição observados em Unidade de Tratamento Intensivo de um hospital do interior de São Paulo, Brasil (outubro de 2014), conforme classificação para os principais medicamentos envolvidos

\begin{tabular}{|c|c|c|c|c|c|c|c|c|c|c|c|c|c|c|c|c|}
\hline \multirow{3}{*}{ Fármaco } & \multicolumn{14}{|c|}{ Erro de diluição } & & \\
\hline & \multicolumn{2}{|c|}{ 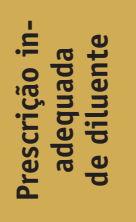 } & \multicolumn{2}{|c|}{ 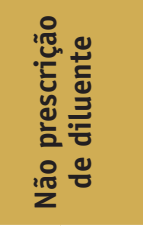 } & \multicolumn{2}{|c|}{ 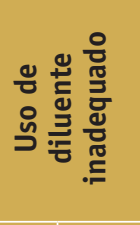 } & \multicolumn{2}{|c|}{ 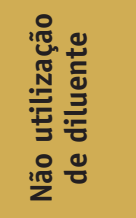 } & \multicolumn{2}{|c|}{ 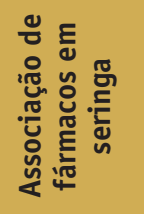 } & \multicolumn{2}{|c|}{ 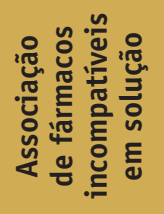 } & \multicolumn{2}{|c|}{ 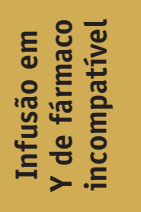 } & \multicolumn{2}{|c|}{ Total } \\
\hline & $n$ & $\%$ & $\mathrm{n}$ & $\%$ & $n$ & $\%$ & $\mathrm{n}$ & $\%$ & $n$ & $\%$ & $\mathrm{~N}$ & $\%$ & $\mathrm{n}$ & $\%$ & $\mathrm{n}$ & $\%$ \\
\hline dipirona & 0 & 0 & 12 & 4,8 & 0 & 0 & 0 & 0 & 4 & 1,6 & 0 & 0 & 12 & 4,8 & 28 & 11,2 \\
\hline fenitoína & 5 & 2 & 5 & 2 & 7 & 2,8 & 0 & 0 & 0 & 0 & 6 & 2,4 & 9 & 3,6 & 32 & 12,8 \\
\hline fentanila & 5 & 2 & 3 & 1,2 & 5 & 2 & 8 & 3,2 & 0 & 0 & 0 & 0 & 7 & 2,8 & 28 & 11,2 \\
\hline furosemida & 0 & 0 & 9 & 3,6 & 0 & 0 & 0 & 0 & 2 & 0,8 & 0 & 0 & 15 & 6 & 26 & 10,4 \\
\hline hidrocortisona & 0 & 0 & 7 & 2,8 & 6 & 2,4 & 0 & 0 & 3 & 1,2 & 0 & 0 & 5 & 2 & 21 & 8,4 \\
\hline metoclopramida & 0 & 0 & 12 & 4,8 & 0 & 0 & 0 & 0 & 9 & 3,6 & 0 & 0 & 7 & 2,8 & 28 & 11,2 \\
\hline midazolam & 0 & 0 & 0 & 0 & 4 & 1,6 & 2 & 0,8 & 0 & 0 & 0 & 0 & 8 & 3,2 & 14 & 5,6 \\
\hline norepinefrina & 13 & 5,2 & 0 & 0 & 15 & 6 & 0 & 0 & 0 & 0 & 0 & 0 & 4 & 1,6 & 32 & 12,8 \\
\hline omeprazol & 7 & 2,8 & 3 & 1,2 & 3 & 1,2 & 0 & 0 & 0 & 0 & 4 & 1,6 & 3 & 1,2 & 20 & 8 \\
\hline ranitidina & 0 & 0 & 9 & 3,6 & 0 & 0 & 0 & 0 & 2 & 0,8 & 0 & 0 & 10 & 4 & 21 & 8,4 \\
\hline Total & 30 & 12 & 60 & 24 & 40 & 16 & 10 & 4 & 20 & 8 & 10 & 4 & 80 & 32 & 250 & 100 \\
\hline
\end{tabular}

O preparo e a administração de medicamentos são uma das atividades mais sérias e de maior responsabilidade da enfermagem e, para sua execução, é necessária a aplicação de vários princípios cientí- ficos associados à existência de um sistema de medicação seguro, com processos desenvolvidos para dificultar as oportunidades de erros, auxiliando o profissional a não errar (3). 
Ao analisar os resultados do presente estudo, foi constatada uma taxa de erro elevada no processo de prescrição, configurada aqui como prescrição inadequada de diluente (12\%) e não prescrição de diluente (24\%), totalizando o porcentual de $36 \%$. Tal resultado comprovam outros achados $(1,2,3,18)$ que definiram este problema como fator causal para a instalação de erro de medicação.

Em relação aos erros de medicação envolvendo o uso de diluente inadequado, foi obtido um índice igual a $16 \%$, superior ao encontrado em um estudo $(9,77 \%)(20)$, e inferior ao descrito por outro estudo $(67,85 \%)$ (21). Contudo, tal fato pode ser justificado pelas diferentes abordagens metodológicas adotadas nestes estudos.

O não uso de diluente (4\%), a associação de medicamentos incompatíveis em seringa (8\%) e soluções (4\%) vêm compor o erro de preparo de medicação, totalizando o porcentual de $16 \%$. Este resultado apresentou taxa inferior à obtida em outro estudo, estimado em $62,69 \%$, o que talvez se justifique pelas diferentes variáveis observadas (1). Contudo, quando o resultado foi comparado com outros estudos, estes apresentaram taxas semelhantes entre si $(2,3,10,22,23)$. Tal resultado pode ser justificado pelo evidenciado em outro estudo, no qual $40,4 \%$ de uma equipe de saúde formada por médicos, enfermeiros, auxiliares e técnicos de enfermagem, e escriturários envolvidos em alguma etapa do processo do sistema de medicação, apresentaram dúvidas quanto à necessidade de diluição e ao diluente a ser usado, à quantidade e validade do medicamento diluído e à possibilidade de precipitação (24).

A incompatibilidade medicamentosa quando ocorre é considerada um erro de medicação, e o produto resultante pode afetar a eficácia e a segurança da terapia, sendo que reconhecer seus fundamentos pode ajudar a prevenir sua ocorrência diminuindo, assim, seus riscos. $\mathrm{O}$ erro envolvendo a infusão em $\mathrm{Y}$ de medicamento incompatível configura um erro de administração medicamentosa, e correspondeu a $32 \%$ dos erros observados neste estudo (25). Este resultado corrobora com outros estudos $(1,2,3,11,20,24)$ e está relacionado aos esquemas terapêuticos envolvendo a prescrição simultânea de múltiplos fármacos intravenosos, às falhas na comunicação entre a equipe de farmácia e a equipe assistencial em fornecer suporte nas questões relacionadas à compatibilidade dos medicamentos; à falha no planejamento assistencial de enfermagem relacionada ao aprazamento de vários fármacos intravenosos; e à necessidade de associar diferentes medicamentos numa única via sem que esta esteja pautada em fundamentos teóricos consistentes.

Em relação aos medicamentos envolvidos nos erros de diluição de medicação, a fenitoína e norepinefrina apresentaram destaque, com índice de erros igual a $12,8 \%$. Na categoria referente à incompatibilidade medicamentosa, estes medicamentos apresentaram taxas inferiores àquelas obtidas em outro estudo, que obteve para a fenitoína, taxa igual a 7,63\%; e para a norepinefrina $6,50 \%$; no presente estudo, as taxas foram de $6 \% \mathrm{e}$ $1,6 \%$ para a fenitoína e norepinefrina, respectivamente (25).

Para a avaliação dos demais parâmetros envolvendo erros de diluição de medicamentos não foram encontrados estudos que contivessem este enfoque. Ao que se refere à busca de informação por parte dos profissionais de saúde envolvidos no sistema de preparo e administração de medicamentos, principalmente, para os medicamentos injetáveis, vale a pena ressaltar que, quando realizada em bula, esta pode representar um problema para a segurança para a farmacoterapia, pois a fonte consultada pode não corresponder ao medicamento em uso, ou seja, a bula e o medicamento em investigação podem não ser do mesmo fabricante. $\mathrm{E}$ ainda que os fármacos sejam correspondentes, as informações contidas na bula não devem ser entendidas como um padrão para a especialidade farmacêutica. $\mathrm{O}$ acervo diferenciado mantido nas unidades hospitalares com diferentes fabricantes, assim como a desvinculação deste medicamento de sua bula no processo de dispensação, colabora para a aquisição inadequada dessas informações em fontes questionáveis. 


\section{Unorana}

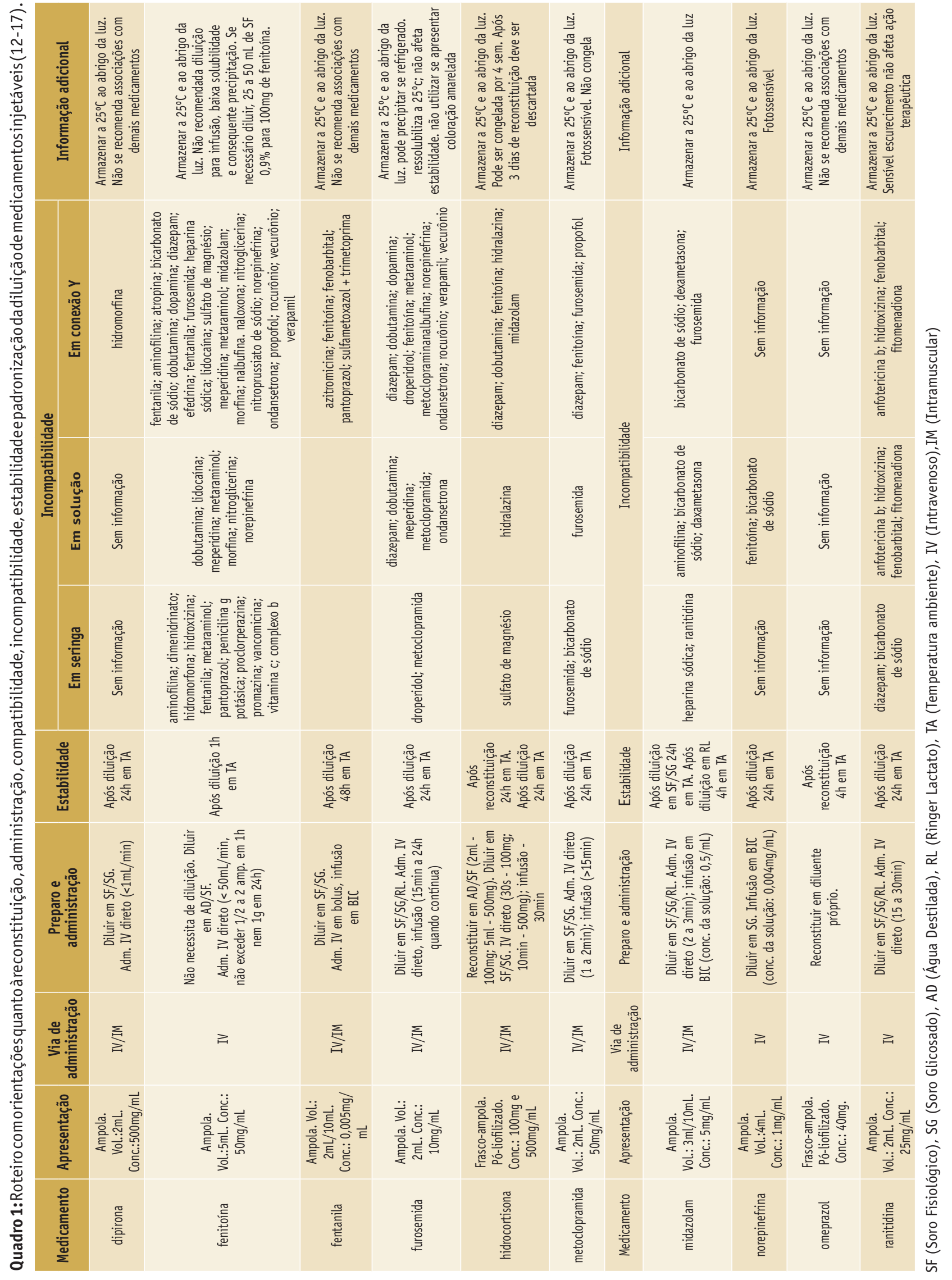




\section{CONCLUSÃO}

Os resultados mostraram uma alta prevalência de erros no processo de medicação em ambiente hospitalar, destacando-se neste estudo os erros de medicação referentes a diluição de medicamentos intravenosos, ratificando dados encontrados na literatura. A frequência de erros de diluição de medicamentos é um risco permanente, principalmente entre pacientes altamente vulneráveis e polimedicados. Nesta perspectiva, profissionais de saúde envolvidos no processo medicamentoso devem averiguar constantemente a medicação a ser administrada aos pacientes para identificar os possíveis erros de medicação e assim contribuir para a segurança

\section{REFERÊNCIAS}

1. Camerini FG, Silva LD. Segurança do paciente: análise do preparo de medicação intravenosa em hospital da rede sentinela. Texto contexto - enferm.2011; 20(1): 41-49. DOI: http://dx.doi.org/10.1590/S010407072011000100005.

2. Silva AEBC, Cassiani SHB. Erros de medicação em hospital universitário: tipo, causas, sugestões e providências. Rev. Bras. Enferm. 2004; 57(6): 671-674. DOI: http://dx.doi.org/10.1590/S0034-71672004000600007.

3. Miasso AI, Grou CR, Cassiani SHB, Silva AEBC, Fakih FT. Erros de medicação: tipos, fatores causais e providências tomadas em quatro hospitais brasileiros. Rev. Esc. Enferm. USP. 2006; 40(4): 524-532. DOI: http:// dx.doi.org/10.1590/S0080-62342006000400011.

4. Anacleto TA, Rosa MB, Neiva HM, Martins, MAP. Erros de medicação. 2010. Pharmacia Brasileira. Disponível em: < http://www.cff.org.br/sistemas/geral/revista/ pdf/124/encarte_farmaciahospitalar.pdf $>$.

5. Ribeiro E. Sistemas de distribuição de medicamentos para pacientes internados. In: Storpitis S. et al. Ciências farmacêuticas: farmácia clínica e atenção farmacêutica. Rio de Janeiro: Guanabara Koogan. 2008. p. 161 - 170.

6. Pereira CC, Gomes FV, Cornélio RCAC, Sousa OV, Alves M. Araújo ALA. Descrição e avaliação do sistema de medicação do serviço de farmácia em um hospital universitário. Lat. Am. J. Pharm. 2009; 28 (1): 91-96.

7. Bohomol E, Ramos LH. Erro de medicação: importância da notificação no gerenciamento da segurança do paciente. Rev. Bras. Enferm. 2007; 60(1): 32-36. doi: http://dx.doi.org/10.1590/S0034-71672007000100006.

8. Coimbra JAH, Cassiani SHB. Segurança na utilização de medicamentos: dificuldades de detecção dos erros de medicação e algumas propostas de prevenção. Ciência, Cuidado e Saúde. 2004; 3(2):153-160. do paciente e o uso racional de medicamentos. A busca do trabalho colaborativo entre estes profissionais de saúde deve ser constante objetivando a intensificação do processo de cuidados em saúde, o compartilhamento de conhecimentos e a integralidade do cuidado. Medidas como treinamento sobre erros de medicação, adoção de relatórios de ocorrência de erros sem consequentes punições aos envolvidos, implementação de medidas administrativas voltadas ao planejamento do sistema de medicação e não aos indivíduos, instalação de serviço de farmácia clínica com planejamento de um sistema de rastreabilidade e prevenção de erros de medicamentos, podem ser estratégias iniciais em busca de maior segurança para o paciente.

9. Santos JO, Silva AEBC, Munari DB, Miasso AI. Condutas adotadas por técnicos de enfermagem após ocorrência de erros de medicação. Acta Paul. Enferm. 2010; 23(3): 328-333. DOI: http://dx.doi.org/10.1590/S010321002010000300003.

10. Melo ABR, Silva LD. Segurança na terapia medicamentosa: uma revisão bibliográfica. Esc. Anna Nery. 2008; 12(1): 166-172. DOI: http://dx.doi.org/10.1590/S141481452008000100026.

11. Cambrussi MC, Hilts LF, Carneiro MB. ncompatibilidade de fármacos não-antineoplásicos administrados através de injeção em Y em pacientes oncológicos. Rev. Bras. Farmacia. 2012; 93(1):85-90.

12. Souza GB. Manual de drogas injetáveis: reconstituição, administração, compatibilidade, incompatibilidade, estabilidade e padronização da diluição de medicamentos injetáveis. São Paulo: Livraria e Editora Medfarma, 2011.

13. Gahart BL, Nazareno AR. Medicamentos intravenosos. 26 ed. Rio de Janeiro: Elsevier, 2011.

14. Schvartsman C, Lewi DS, Morgulis RNF, Almeida SM. Manual Farmacêutico. 14ed. São Paulo, Hospital Albert Einstein, 2010. Disponível em: $<$ http://medsv1.einstein. br/diretrizes/farmacia/ManualFarm11.pdf $>$.

15. Melo VV, Duarte IP, Soares AQ. Guia de Estabilidade de Medicamentos Injetáveis. 1. ed. - (3 Atualização) - Goiânia, 2012. Disponível em: <https://hc.ufg.br/ up/138/o/Guia_de_Estabilidade_de_Medicamentos. pdf $>$.

16. Oliveira HC, Negrão NY M. I Guia prático de incompatibilidades entre os principais medicamentos utilizados na unidade de terapia intensiva do HUJM. Cuiabá, 2012. Disponível em: < http://www.ufmt.br/hujm/arquivos/6a9 1853a69434c9e3ae1790f23ba7229.pdf $>$ 
17. Machado E, Ricieri MC, Perozin MM. Manual de administração de medicamentos injetáveis. 2012. Disponível em: <http://www.sbrafh.org.br/site/public/ temp/503cb71ad536d.pdf $>$.

18. Padilha KG, Kitahara PH, Gonçalves CCS, Sanches ALC. Ocorrências iatrogênicas com medicação em Unidade de Terapia Intensiva: condutas adotadas e sentimentos expressos pelos enfermeiros. Rev Esc Enferm USP. 2002; 36(1): 50-7. DOI: http://dx.doi.org/10.1590/ S0080-62342002000100008.

19. Lopes MJM; Leal SMC. A feminização persistente na qualificação profissional da enfermagem brasileira. Cad. Pagu. 2005; 24(1): 105-125. DOI: http://dx.doi. org/10.1590/S0104-83332005000100006.

20. Miranda TMM, Petriccione S, Ferracini FT, Borges FWM. Intervenções realizadas pelo farmacêutico clínico na unidade de primeiro atendimento. Einstein (São Paulo).2012; 10(1): 74-78. DOI: http://dx.doi.org/10.1590/ S1679-45082012000100015.

21. Lisboa CD, Silva LD, Matos GC. Investigação da técnica de preparo de medicamentos para administração por cateteres pela enfermagem na terapia intensiva.
Rev. Esc. Enferm. USP. 2013; 47(1): 53-60. DOI: http:// dx.doi.org/10.1590/S0080-62342013000100007.

22. Grou CR, Cassiani SHB, Telles Filho PCP, Opitz SP. Conhecimento de enfermeiras e técnicos de enfermagem em relação ao preparo e administração de medicamentos. Einstein. 2004. 2(3):182-186.

23. Miasso AI, Silva AEBC, Cassiani SHB, Grou CR, Oliveira RC, Fakih F. O processo de preparo e administração de medicamentos: identificação de problemas para propor melhorias e prevenir erros de medicação. Rev. Lat-Am. Enferm. 2006; 14(3): 354-363. DOI: http:// dx.doi.org/10.1590/S0104-11692006000300008.

24. Silva DO, Grou CR, Miasso AI, Cassiani SHB. Preparo e administração de medicamentos: análise de questionamentos e informações da equipe de enfermagem. Rev Lat-Am Enferm. 2007; 15(5).

25. Moraes CG. Análise de incompatibilidade de medicamentos intravenosos no centro de tratamento intensivo adulto do hospital de clínicas de Porto Alegre [trabalho de conclusão de curso]. Porto Alegre: Universidade Federal do Rio Grande do Sul. Curso de Farmácia; 2010. 